\title{
The completeness of the fossil record of plesiosaurs, marine reptiles from the Mesozoic
}

Samuel L. Tutin and Richard J. Butler

Acta Palaeontologica Polonica 62 (3), 2017: 563-573 doi:https://doi.org/10.4202/app.00355.2017

Plesiosaurs were a highly successful group of marine reptiles occurring worldwide in the Jurassic and Cretaceous, but to date few studies have focused on their preservation through time. Here, we conduct the first detailed assessment of the quality of the plesiosaur fossil record. Data was compiled for 178 specimens representing 114 valid species. For each species we calculated the character completeness metric (CCM: percentage of phylogenetic characters from a cladistic dataset that can be scored for that species) and the skeletal completeness metric (SCM: percentage of the overall skeleton that is preserved for that species). Average CCM and SCM values were calculated for individual geological stages. A strong significant positive correlation was recovered between CCM and SCM, suggesting that the two metrics are recording the same signal, at least for this clade. Although a significant correlation between changes in sea level and changes in plesiosaur completeness was not recovered, an underlying negative relationship may be present but obscured by poorly sampled time bins. Plesiosaur completeness though time is not significantly correlated with that for contemporary terrestrial groups (sauropods, pterosaurs, birds), but is significantly correlated with that for ichthyosaurs, suggesting common controls on skeletal preservation in the marine realm. Significantly higher median completeness values in plesiosaurs and ichthyosaurs than in contemporary terrestrial groups support the hypothesis that the marine tetrapod fossil record is more complete than that of terrestrial tetrapods. A collector's curve for plesiosaurs shows a generally slow constant rate of discovery from the latter part of the 19th century until the 1990s, at which point the rate of discovery increased substantially and shows no sign of slowing. A significant but very weak negative correlation between SCM and the year in which a taxon was named suggests a weak tendency for more recently named species to have less complete skeletons.

Key words: Plesiosauria, Ichthyosauria, diversity, macroevolution, sea level, Jurassic, Cretaceous.

Samuel Tutin [samtutin@icloud.com] and Richard J. Butler [r.butler.1@bham.ac.uk] (corresponding author), School of Geography, Earth and Environmental Sciences, University of Birmingham, Edgbaston, Birmingham, B15 2TT, UK. 
This is an open-access article distributed under the terms of the Creative Commons

Attribution License (for details please see creativecommons.org), which permits unrestricted use, distribution, and reproduction in any medium, provided the original author and source are credited.

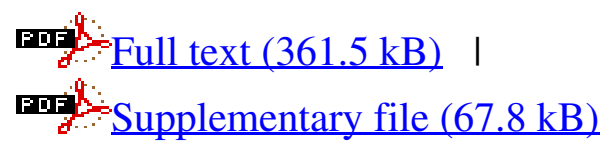

